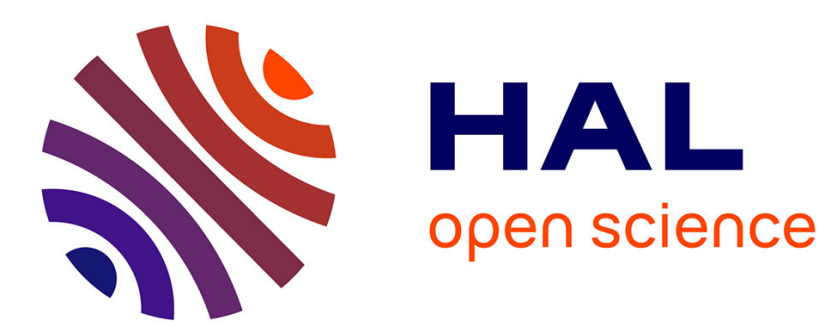

\title{
Fragmented Superradiance of a Bose-Einstein Condensate in an Optical Cavity
}

\author{
Axel Lode, Christoph Bruder
}

\section{To cite this version:}

Axel Lode, Christoph Bruder. Fragmented Superradiance of a Bose-Einstein Condensate in an Optical Cavity. Physical Review Letters, 2017, 118, pp.013603. 10.1103/PhysRevLett.118.013603 . hal02370009

\section{HAL Id: hal-02370009 \\ https://hal.science/hal-02370009}

Submitted on 19 Nov 2019

HAL is a multi-disciplinary open access archive for the deposit and dissemination of scientific research documents, whether they are published or not. The documents may come from teaching and research institutions in France or abroad, or from public or private research centers.
L'archive ouverte pluridisciplinaire HAL, est destinée au dépôt et à la diffusion de documents scientifiques de niveau recherche, publiés ou non, émanant des établissements d'enseignement et de recherche français ou étrangers, des laboratoires publics ou privés. 


\title{
Fragmented Superradiance of a Bose-Einstein Condensate in an Optical Cavity
}

\author{
Axel U. J. Lode ${ }^{*}$ and Christoph Bruder \\ Department of Physics, University of Basel, Klingelbergstrasse 82, CH-4056 Basel, Switzerland
}

(Received 9 June 2016; revised manuscript received 29 September 2016; published 6 January 2017)

\begin{abstract}
The Dicke model and the superradiance of two-level systems in a radiation field have many applications. Recently, a Dicke quantum phase transition has been realized with a Bose-Einstein condensate in a cavity. We numerically solve the many-body Schrödinger equation and study correlations in the ground state of interacting bosons in a cavity as a function of the strength of a driving laser. Beyond a critical strength, the bosons occupy multiple modes macroscopically while remaining superradiant. This fragmented superradiance can be detected by analyzing the variance of single-shot measurements.
\end{abstract}

DOI: 10.1103/PhysRevLett.118.013603

The Dicke quantum phase transition from a normal to a superradiant state is driven by the cooperativity of the emitters in a light field [1-4]. It has recently been realized and studied in a number of different systems like superconducting qubits coupled to resonators [5-8], electromagnetically coupled quantum dots [9], the magnetoresistance of organic light-emitting diodes [10], a plasma of Helium atoms [11], a superradiant laser [12], and a Bose-Einstein condensate in an optical cavity [13-17]. All of these systems are formed of constituents which are more complex than the two-level emitters considered in the Dicke model which makes its successful application to these systems even more remarkable.

The unique experimental control of Bose-Einstein condensates of ultracold atoms [18-20] has made them versatile quantum simulators for other systems, like here, the Dicke Hamiltonian [13-16,21]. Bose-Einstein condensates or ultracold Fermi gases, however, cannot generally be described within a two-level framework since they constitute many-body systems of interacting atoms [22-25]. In many-body systems, correlations arise due to the interactions between the particles, and hence, two-level descriptions [26,27] or mean-field approaches [28-30] might fail to accurately describe them [24,25,31,32]. One striking example for a correlation effect not captured by mean-field methods is the emergence of fragmentation [33-35] in interacting Bose-Einstein condensed systems: the reduced one-body density matrix starts to have more than one macroscopic eigenvalue.

In this Letter, we will focus on a Bose-Einstein condensate in an optical cavity and show how the fact that the Bose-Einstein condensate is an interacting manybody system and not just an ensemble of two-level systems substantially enriches the phase diagram beyond the Dicke model. Our focus, here, is on the emergent phases triggered by interactions in a zero-temperature system. The phase diagram of the noninteracting system at finite temperature (the Dicke-Hepp-Lieb phase transition) is discussed in Ref. [36].
In the following, we apply a numerical many-body approach for ultracold atoms in multimodal cavities and demonstrate that their ground state in a single-mode cavity exhibits correlations that indicate the fragmentation of the system. For sufficient pump power, the system enters a state of fragmented superradiance. The pump power needed to trigger the emergence of correlations in the ground state of the atoms is generally larger than the pump power necessary to enter the superradiant state. The existence of this third phase modifies the phase diagram of ultracold bosons in an optical cavity and demonstrates the limitations of the mapping of the system to the Dicke model which exhibits only two phases for bosons in single-mode cavities.

The realization of the Dicke quantum phase transition with a Bose-Einstein condensate in a single-mode optical cavity in [13-16] motivates us to study the role of correlations in the process of self-organization. To proceed, we investigate the ground state of a system of $N=100$ interacting bosonic atoms in a single-mode cavity as a function of the pump rate, see Fig. 1 for a scheme of the system.
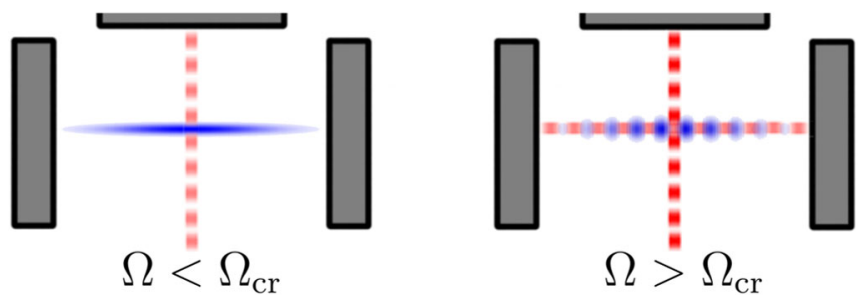

FIG. 1. Setup for the Dicke quantum phase transition with a Bose-Einstein condensate in an optical cavity. For a cavity pump power $\Omega$ that is smaller than the critical pump power $\Omega_{\mathrm{cr}}$, the Bose-Einstein condensate (blue) in the cavity formed by the left and right mirrors (gray) is unaffected by the pump laser and no population of cavity photons (red) is built up (left panel). For pump powers $\Omega$ larger than the critical value $\Omega_{\mathrm{cr}}$, the BoseEinstein condensate self-organizes as a consequence of the potential which is built up by the population of the cavity with photons (right panel). 
The bosons are governed by the time-dependent manybody Schrödinger equation $i \partial_{t}|\Psi\rangle=H|\Psi\rangle$ with the Hamiltonian

$$
H=\sum_{i=1}^{N}\left[T_{\mathbf{r}_{i}}+V\left(\mathbf{r}_{i}\right)\right]+\lambda_{0} \sum_{i>j=1}^{N} \delta\left(\mathbf{r}_{i}, \mathbf{r}_{j}\right)
$$

Here, $T_{\mathbf{r}_{i}}$ is the kinetic energy of the $i$ th atom, $V(\mathbf{r})=$ $V_{\text {1-body }}(\mathbf{r})+V_{\text {cavity }}(\mathbf{r})$ is a one-particle potential that contains the external trapping $V_{1-\text { body }}$ of the atoms and the potential $V_{\text {cavity }}$ generated by the photons in the cavity and pump laser beam (see right panel of Fig. 1). The terms proportional to $\lambda_{0}$ generate a repulsive contact interaction. We choose $\lambda_{0}=0.01$ for the interaction strength $\lambda_{0}$ which is proportional to the $s$-wave scattering length and adjustable in experiments (see Ref. [37] for experimental parameters to realize this choice for $\lambda_{0}$ ). The potential $V_{\text {cavity }}(\mathbf{r})$ is a function of the cavity field amplitude $\alpha$, which is in turn obtained from the equation of motion of the cavity $[30,39]$,

$$
\begin{aligned}
i \partial_{t} \alpha(t)= & \left(-\Delta_{c}+\sum_{k, q=1}^{M} \rho_{k q}(t) U_{k q}(t)-i \kappa\right) \alpha(t) \\
& +\sum_{k, q=1}^{M} \rho_{k q}(t) \eta_{k q}^{d}(t) .
\end{aligned}
$$

Here, we used $\langle\Psi(t)|U(\mathbf{r})| \Psi(t)\rangle=\sum_{k, q=1}^{M} \rho_{k q}(t) U_{k q}(t)$ and $\langle\Psi(t)|\eta(\mathbf{r}, t)| \Psi(t)\rangle=\sum_{k, q=1}^{M} \rho_{k q}(t) \eta_{k q}(t)$, where $U(\mathbf{r})$ and $\eta(\mathbf{r}, t)$ are proportional to the cavity mode and pump laser profiles, and $\rho_{k q}$ are the matrix elements of the reduced one-body density matrix that is normalized to $N$, see [39]. The cavity is detuned by $\Delta_{c}$ from the atomic resonance and its loss rate is given by $\kappa$. We employ the multiconfigurational time-dependent Hartree method for indistinguishable particles (MCTDH-X) to compute the ground state of the many-body system coupled to the equation of motion of the cavity amplitude, see Supplemental Material [39], as well as Refs. [24,32,4143], for details.

In the following, we will consider a one-dimensional setup, i.e., a collinear arrangement of the pump laser and the trapped atoms, and use the coordinate $x$ instead of $\mathbf{r}$. We assume the external confinement to be harmonic, $V_{1-\text { body }}(x)=\frac{1}{2} x^{2}$ and choose dimensionless units [37] and cavity parameters $[39,44]$. The potential exerted on the bosons by the photons in the pump laser and the cavity $[13,39]$ is given by

$$
V_{\text {cavity }}(x)=|\alpha|^{2} U_{0} \cos ^{2}(k x)+\left(\alpha+\alpha^{*}\right) \eta \cos (k x) .
$$

Here, the terms proportional to $U_{0}$ and $\eta$ refer to the cavity photons and the pump laser, respectively, see Eq. (2) and the Supplemental Material [39].
Since our system is one-dimensional and parabolically confined, we expect to discover physics different from previous investigations in two-dimensional systems in a lattice, where superfluid self-organized and Mott-insulator self-organized [45] as well as supersolid and charge-density wave $[46,47]$ phases have been demonstrated.

As a first step in our investigation, we show the density $\rho(x)$ and the one-body potential $V(x)$ of the ground state as a function of the cavity pump power in Fig. 2. Beyond the critical pump power $\Omega_{\mathrm{cr}}$, the cavity population $|\alpha|^{2}$ rises, see Fig. 3(a), and is roughly proportional to the magnitude of the maxima of the potential shown in the lower panel of

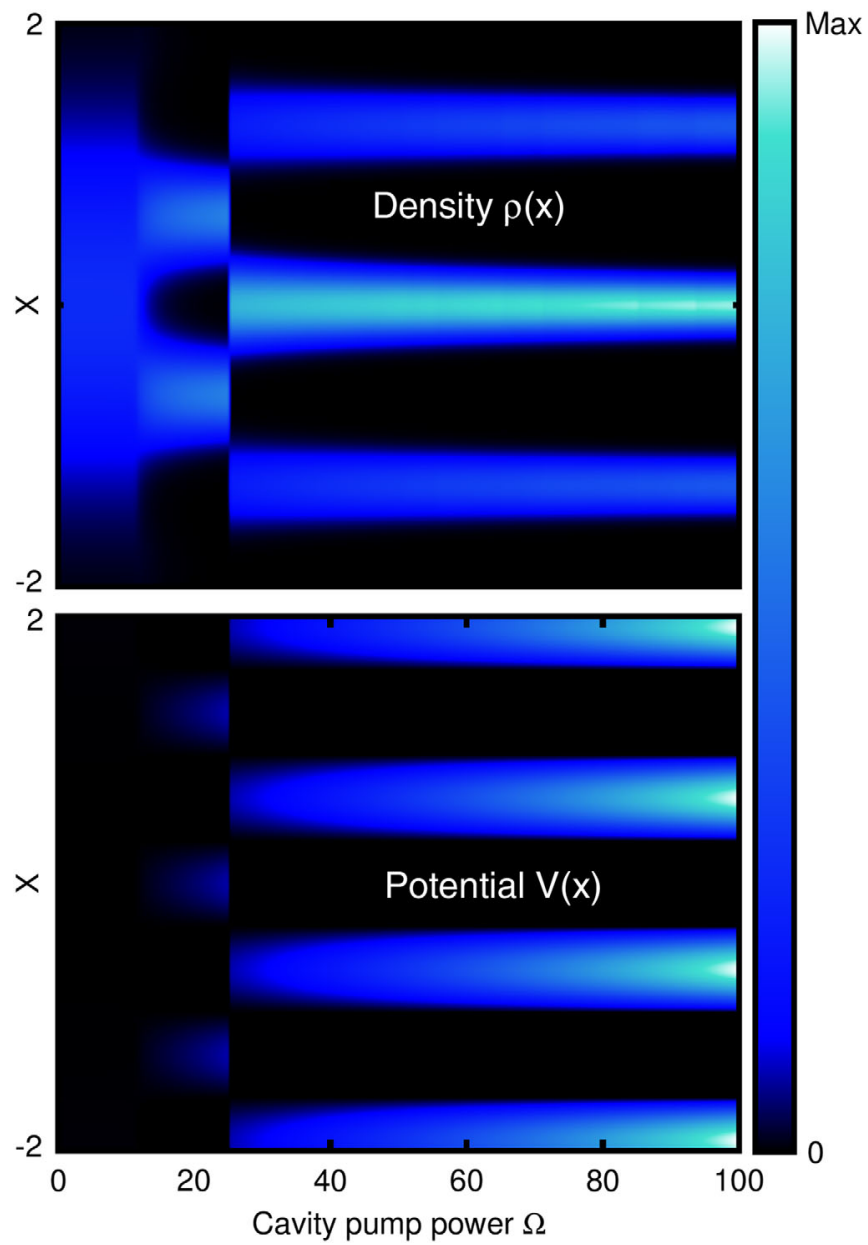

FIG. 2. Self-organization of the ground state of a Bose-Einstein condensate. The density $\rho(x)$ of the atoms and the potential $V(x)=V_{1 \text {-body }}(x)+V_{\text {cavity }}(x)$ is shown as a function of the cavity pump power in the upper and lower panels, respectively. Once the applied pump power exceeds a critical value, the atoms self-organize because the field which is built up inside the cavity creates a periodic one-body potential (cf. lower and upper panels). As a result of a competition between external and cavity potential as well as interactions, the sign of the cavity amplitude switches one time for $\Omega \approx 25$ [compare pattern in density and potential with the inset of Fig. 3(a)]. All quantities shown are dimensionless, see text for further discussion. 
Fig. 2. The density becomes self-organized and the atoms cluster around the minima of the cavity-photon-mediated potential $V_{\text {cavity }}$ (compare upper and lower panels in Fig. 2) instead of the minimum of the parabolic confinement $V_{\text {1-body }}(x)$. This self-organization of the atoms marks the transition to the superradiant phase of the Dicke model $[15,16]$. The emergent density resembles the density of atoms in optical lattices.

To assess whether or not correlations between the atoms are built up in their self-organization process, we investigate the cavity population and fragmentation of the system as a function of the cavity pump power. We quantify the fragmentation using the fraction $F$ of atoms which do not occupy the lowest eigenstate of the reduced one-body density matrix

$$
\rho^{(1)}\left(x, x^{\prime}\right)=\sum_{k} \rho_{k}^{(\mathrm{NO})} \phi_{k}^{(\mathrm{NO}), *}(x) \phi_{k}^{(\mathrm{NO})}\left(x^{\prime}\right) .
$$

The eigenvalues $\rho_{k}^{(\mathrm{NO})}$ and eigenfunctions $\phi_{k}^{(\mathrm{NO})}(x)$ of $\rho^{(1)}$ are known as natural occupations and natural orbitals, respectively [superscript (NO)]. If $\rho^{(1)}$ has only a single macroscopic eigenvalue, $F=0$, the corresponding system is referred to as condensed [48]. If, on the contrary, $\rho^{(1)}$ has multiple macroscopic eigenvalues, $F>0$, the corresponding system is referred to as fragmented [33-35]. Figures 3(b) and 3(c) show a plot of the excited fraction $F$ and the eigenvalues $\rho_{k}^{(\mathrm{NO})}$, respectively. At pump powers $\Omega \gtrsim 40>\Omega_{\mathrm{cr}}$, i.e., well into the superradiant regime, the system fragments. This transition to fragmentation indicates the emergence of correlations and marks the breakdown of mean-field approaches like the Gross-Pitaevskii equation, which cannot capture correlations and fragmentation [25,31]. Furthermore, the simple two-level description of the superradiant system ceases to be applicable for the fragmented superradiant system, see Sec. IV of [39] for details.

The occurrence of this fragmented superradiant phase is one of the main results of our Letter. This result is robust against variations in the particle number as demonstrated by a finite-size scaling plot, see inset in Fig. 3(c). Now, we discuss how the predicted fragmentation may be detected experimentally. The emergent phase cannot be detected in the photonic part of the system alone, see Fig. 3(a). Its detection requires the simultaneous analysis of the wave function of the atoms in the cavity and the cavity photons. We have found that the fragmentation of the Bose-Einstein condensate can be detected in the variance $\mathcal{V}$ of single-shot measurements [39,49] of the momentum distribution, see Fig. 3(d) for a plot of $\mathcal{V}$. This variance maps the excited fraction of atoms accurately and can, therefore, be used to assess the fragmentation of the system, compare Figs. 3(b) and $3(\mathrm{~d})$.

The similarity of the behavior of the excited fraction $F$ and the single-shot variance $\mathcal{V}$ may be understood qualitatively: For a coherent condensate, $F \approx 0$, the variance $\mathcal{V}$ is

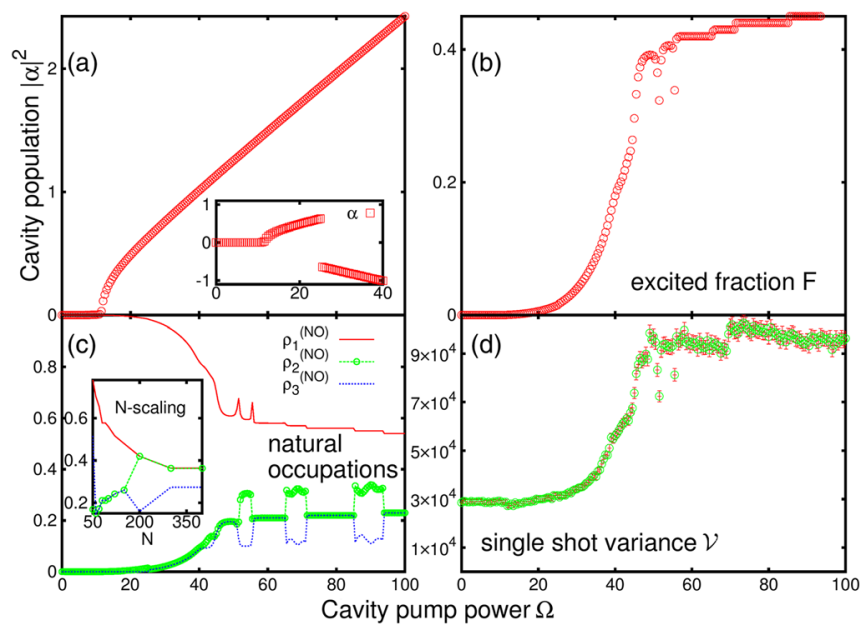

FIG. 3. (a) Buildup of cavity population in the self-organization process. Beyond the critical value $\Omega_{\mathrm{cr}} \approx 12$ the population of photons in the cavity grows with increasing pump power $\Omega$. The cavity population $|\alpha|^{2}$ drives the self-organization of the atoms, see Eq. (3). Inset: The amplitude $\alpha$ shows a sign change from a positive to a negative value in the superradiant phase at $\Omega \approx 25$ and continues to decrease monotonically in the fragmented superradiant phase. (b) Fraction $F$ of atoms that are outside the natural orbital with the largest occupation, (c) natural orbital occupations, and (d) variance $\mathcal{V}$ in single shots of the momentum density (1000 samples per data point), all as a function of the pump power $\Omega$. The variance $\mathcal{V}$ maps the fraction $F$ of atoms in excited orbitals closely, compare panels (b) and (d). The natural occupations (c) show that the dips in the excited fraction $F$ and the variance are due to a redistribution of atoms between excited orbitals. The inset of (c) demonstrates that fragmented superradiance emerges for a range of particle numbers $N$ with a finitesize scaling for $\Omega=80$. The emergence of fragmentation and the growth of $F$ signals that the system enters a new phase with many-body correlations between the atoms. This is confirmed by comparing (b) and (c) with Figs. 4(a)-4(c). All quantities shown are dimensionless, see text.

minimized because all the atoms in the respective singleshot measurements are picked from the same natural orbital. For a fragmented state, $F>0$, the variance of the single shots grows, since the atoms are picked from a superposition of several mutually orthonormal natural orbitals $\phi_{1}^{(\mathrm{NO})}, \phi_{2}^{(\mathrm{NO})}, \ldots$. The momenta obtained by drawing from this distribution that lives in a larger space spanned by several orbitals have a wider spread. Hence, the variance $\mathcal{V}$ is larger as compared to the values obtained by drawing momenta from a single orbital. We expect that the (momentum-space) variance of single-shot measurements can also be used to quantify fragmentation experimentally in more general setups. Since single-shot measurements require only absorption images, this would mark a clear advantage in comparison to other methods of determining fragmentation that require the measurement of the off-diagonal part of the reduced one-body density matrix [33-35] or density-density correlations [50]. 


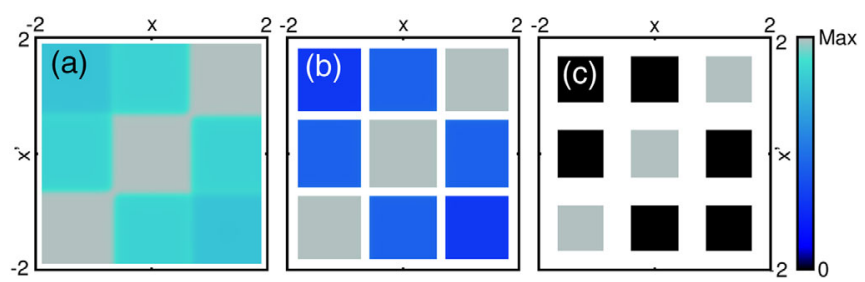

FIG. 4. Tracing the transition from superradiance to fragmented superradiance in the spatial correlation function. The correlation function $\left|g^{(1)}\left(x, x^{\prime}\right)\right|^{2}$ in the superradiant phase is shown for pump powers (a) $\Omega=30$, (b) $\Omega=40$, and (c) $\Omega=100$, wherever the density exceeds 0.001 . With increasing pump power, the atoms in distinct wells gradually loose coherence $\left[\left|g^{(1)}\left(x, x^{\prime} \neq x\right)\right|^{2}\right.$ drops to zero], compare the position of black squares with the upper panel of Fig. 2. The structure of $\left|g^{(1)}\right|^{2}$ in (c) demonstrates the threefold fragmentation in the system. All quantities shown are dimensionless.

To get a detailed picture of the structure of the emergent many-body correlations, we analyze the spatial correlation function $g^{(1)}\left(x, x^{\prime}\right)=\left[\rho^{(1)}\left(x, x^{\prime}\right) / \sqrt{\rho(x) \rho\left(x^{\prime}\right)}\right]$. For a complementary analysis of the momentum correlation function, see [39]. The correlation function $g^{(1)}$ uses the reduced onebody density matrix $\rho^{(1)}$ to measure the proximity of a manybody state to a product or mean-field state for a given set of coordinates $x, x^{\prime}$. It is a key measure for the coherence of ultracold bosonic atoms and is experimentally detectable [51,52]. Figure 4 shows a plot of $\left|g^{(1)}\left(x, x^{\prime}\right)\right|^{2}$. The spatial correlation function gives an intuitive picture of the mechanism behind the fragmentation of the system. The potential exerted on the atoms by the photons in the cavity grows with the pump power. Because of the repulsive interparticle interactions, the atoms in the distinct wells of this cavity-photon-mediated periodic potential become disconnected beyond a critical magnitude of the pump power. Consequently, the spatial correlation function vanishes gradually for off-diagonal values, see Fig. 4. This observation is consistent with the occurrence of fragmentation, see Figs. 3(b) and 3(c). The loss of spatial coherence between atoms in distinct wells of the potential generated by the photons in the cavity is complemented by a periodic pattern of correlated and uncorrelated momenta in the momentum correlation function that reflects the periodicity of the potential, see Supplemental Material [39] and Fig. S1 therein.

In conclusion, we have derived and applied a new numerical many-body approach to describe laser-pumped ultracold atoms in cavity fields. We have used the multiconfigurational time-dependent Hartree method for indistinguishable particles, MCTDH-X, to solve the many-body Schrödinger equation of the coupled cavity-atom system. This method self- and size-consistently incorporates correlations between the atoms in the cavity and can also be applied to multimode cavities and fermionic atoms.

We have demonstrated that the phase diagram of ultracold bosonic atoms in a single-mode cavity exhibits a fragmented superradiant phase in which the atoms show many-body correlations not captured within two-level or mean-field approaches. While the system remains superradiant, the Bose-Einstein condensate starts to macroscopically occupy multiple single-particle states when the pump power is increased sufficiently. Thus, our work adds a new phase to the phase diagram of bosons in a cavity which can be detected using the statistics of experimental single shot measurements and the cavity population.

We would like to thank E. Fasshauer, R. Landig, and M. C. Tsatsos for interesting discussions and, in particular, R. Chitra, P. Molignini, and L. Papariello for pointing out an inconsistency in the normalization of the reduced one-body density matrix. Financial support by the Swiss SNF and the NCCR Quantum Science and Technology as well as computation time on the Hazel Hen supercomputer of the HLRS Stuttgart is gratefully acknowledged.

*axel.lode@unibas.ch

[1] K. Rzaźewski, K. Wódkiewicz, and W. Źakowicz, Phys. Rev. Lett. 35, 432 (1975).

[2] R. H. Dicke, Phys. Rev. 93, 99 (1954).

[3] C. Emary and T. Brandes, Phys. Rev. E 67, 066203 (2003).

[4] H. Ritsch, P. Domokos, F. Brennecke, and T. Esslinger, Rev. Mod. Phys. 85, 553 (2013).

[5] J. A. Mlynek, A. A. Abdumalikov, C. Eichler, and A. Wallraff, Nat. Commun. 5, 5186 (2014).

[6] M. Feng, Y. P. Zhong, T. Liu, L. L. Yan, W. L. Yang, J. Twamley, and H. Wang, Nat. Commun. 6, 7111 (2015).

[7] O. Viehmann, J. von Delft, and F. Marquardt, Phys. Rev. Lett. 107, 113602 (2011).

[8] C. Ciuti and P. Nataf, Phys. Rev. Lett. 109, 179301 (2012).

[9] M. Scheibner, T. Schmidt, L. Worschech, A. Forchel, G. Bacher, T. Passow, and D. Hommel, Nat. Phys. 3, 106 (2007).

[10] D. P. Waters, G. Joshi, M. Kavand, M. E. Limes, H. Malissa, P. L. Burn, J. M. Lupton, and C. Boehme, Nat. Phys. 11, 910 (2015).

[11] H. Xia, A. A. Svidzinsky, L. Yuan, C. Lu, S. Suckewer, and M. O. Scully, Phys. Rev. Lett. 109, 093604 (2012).

[12] J. G. Bohnet, Z. Chen, J. M. Weiner, D. Meiser, M. J. Holland, and J. K. Thompson, Nature (London) 484, 78 (2012).

[13] F. Brennecke, R. Mottl, K. Baumann, R. Landig, T. Donner, and T. Esslinger, Proc. Natl. Acad. Sci. U.S.A. 110, 11763 (2013).

[14] F. Brennecke, T. Donner, S. Ritter, T. Bourdel, M. Köhl, and T. Esslinger, Nature (London) 450, 268 (2007).

[15] K. Baumann, C. Guerlin, F. Brennecke, and T. Esslinger, Nature (London) 464, 1301 (2010).

[16] J. Klinder, H. Keler, M. Wolke, L. Mathey, and A. Hemmerich, Proc. Natl. Acad. Sci. U.S.A. 112, 3290 (2015).

[17] R. Chitra and O. Zilberberg, Phys. Rev. A 92, 023815 (2015).

[18] M. H. Anderson, J. R. Ensher, M. R. Matthews, C. E. Wiemann, and E. A. Cornell, Science 269, 198 (1995). 
[19] C. C. Bradley, C. A. Sackett, J. J. Tollett, and R. G. Hulet, Phys. Rev. Lett. 75, 1687 (1995).

[20] K. B. Davis, M.-O. Mewes, M. R. Andrews, N. J. van Druten, D. S. Durfee, D. M. Kurn, and W. Ketterle, Phys. Rev. Lett. 75, 3969 (1995).

[21] K. Baumann, Ph.D. thesis, ETH Zürich, 2012.

[22] D. Jaksch, C. Bruder, J. I. Cirac, C. W. Gardiner, and P. Zoller, Phys. Rev. Lett. 81, 3108 (1998).

[23] M. Greiner, O. Mandel, T. Esslinger, T. W. Hänsch, and I. Bloch, Nature (London) 415, 39 (2002).

[24] E. Fasshauer and A. U. J. Lode, Phys. Rev. A 93, 033635 (2016).

[25] A. U. J. Lode, K. Sakmann, O. E. Alon, L. S. Cederbaum, and A. I. Streltsov, Phys. Rev. A 86, 063606 (2012).

[26] D. Nagy, G. Kónya, G. Szirmai, and P. Domokos, Phys. Rev. Lett. 104, 130401 (2010).

[27] M. Paternostro, G. De Chiara, and G. M. Palma, Phys. Rev. Lett. 104, 243602 (2010).

[28] C. J. Pethick and H. Smith, Bose-Einstein Condensation in Dilute Gases (Cambridge University Press, Cambridge, England, 2002).

[29] L. P. Pitaevskii and S. Stringari, Bose-Einstein Condensation (Clarendon Press, Oxford, 2003).

[30] D. Nagy, G. Szirmai, and P. Domokos, Eur. Phys. J. D 48, 127 (2008).

[31] A. U. J. Lode, Tunneling Dynamics in Open Ultracold Bosonic Systems, Springer Theses, (Springer, Heidelberg, 2014).

[32] O. E. Alon, A. I. Streltsov, and L. S. Cederbaum, Phys. Rev. A 77, 033613 (2008).

[33] R. W. Spekkens and J. E. Sipe, Phys. Rev. A 59, 3868 (1999).

[34] A. I. Streltsov, O. E. Alon, and L. S. Cederbaum, Phys. Rev. Lett. 99, 030402 (2007).

[35] P. Bader and U. R. Fischer, Phys. Rev. Lett. 103, 060402 (2009).

[36] F. Piazza, P. Strack, and W. Zwerger, Ann. Phys. (Amsterdam) 339, 135 (2013).

[37] We first choose a length scale of $L=1 \mu \mathrm{m}$. The scale of energy for the mass of ${ }^{87} \mathrm{Rb}$ is $\hbar^{2} /\left(m L^{2}\right)=2 \pi \hbar \times 116 \mathrm{~Hz}$, and the scale of time is $m L^{2} / \hbar=1.37 \mathrm{~ms}$. The onedimensional scattering parameter $\lambda_{0}$ is related to the three-dimensional scattering length $a_{3 D}$ by $\lambda_{0}=$ $2 L m \omega_{\perp} a_{3 D} / \hbar$ where $\omega_{\perp}$ is the frequency of the transversal confinement [38]. Using $a_{3 D}=100.4 a_{0}$, where $a_{0}$ is the Bohr radius, and $\lambda_{0}=0.01$, one obtains $\omega_{\perp}=687.9 \mathrm{~Hz}$.

[38] M. Olshanii, Phys. Rev. Lett. 81, 938 (1998).

[39] See Supplemental Material at http://link.aps.org/ supplemental/10.1103/PhysRevLett.118.013603, which includes Ref. [40], for a description of MCTDH-X and its coupling to multi-mode cavities, the measurement of fragmented superradiance using single shots, the effects of fragmented superradiance on the momentum correlation function, and the breakdown of the two-level description in momentum space.

[40] H.-D. Meyer, U. Manthe, and L. S. Cederbaum, Chem. Phys. Lett. 165, 73 (1990); U. Manthe, H.-D. Meyer, and L. S. Cederbaum, J. Chem. Phys. 97, 3199 (1992).

[41] A. U. J. Lode, Phys. Rev. A 93, 063601 (2016).

[42] A. U. J. Lode, M. C. Tsatsos, and E. Fasshauer, мстDH-X, http://ultracold.org; http://mctdh.bf; http://mctdhx.org, 2016.

[43] O. E. Alon, A. I. Streltsov, and L. S. Cederbaum, J. Chem. Phys. 127, 154103 (2007).

[44] In the units of Ref. [37], the cavity detuning is $\Delta_{c}=43$, the cavity loss rate $\kappa=11.2$, and the cavity-atom coupling $g_{0}=46$. The cavity and pump mode functions are given by $\cos \left(k_{c} x\right)$, where the $k_{c}$ value of the cavity is $k_{c}=4.9$.

[45] M. R. Bakhtiari, A. Hemmerich, H. Ritsch, and M. Thorwart, Phys. Rev. Lett. 114, 123601 (2015).

[46] R. Landig, L. Hruby, N. Dogra, M. Landini, R. Mottl, T. Donner, and T. Esslinger, Nature (London) 532, 476 (2016).

[47] Y. Li, L. He, and W. Hofstetter, Phys. Rev. A 87, 051604 (2013).

[48] O. Penrose and L. Onsager, Phys. Rev. 104, 576 (1956).

[49] K. Sakmann and M. Kasevich, Nat. Phys. 12, 451 (2016).

[50] M. K. Kang and U. R. Fischer, Phys. Rev. Lett. 113, 140404 (2014).

[51] T. Betz, S. Manz, R. Bücker, T. Berrada, Ch. Koller, G. Kazakov, I. E. Mazets, H.-P. Stimming, A. Perrin, T. Schumm, and J. Schmiedmayer, Phys. Rev. Lett. 106, 020407 (2011).

[52] A. Perrin, R. Bücker, S. Manz, T. Betz, C. Koller, T. Plisson, T. Schumm, and J. Schmiedmayer, Nat. Phys. 8, 195 (2012). 